\title{
Acknowledgment to Reviewers of Axioms in 2020
}

\author{
Axioms Editorial Office
}

Citation: Axioms Editorial Office.

Acknowledgment to Reviewers of

Axioms in 2020. Axioms 2021, 10, 13 https://doi.org/10.3390/axioms1001 0013

Published: 21 January 2021

Publisher's Note: MDPI stays neutral with regard to jurisdictional claims in published maps and institutional affiliations.

\section{(c) (i)}

Copyright: $@ 2021$ by the author. Licensee MDPI, Basel, Switzerland. This article is an open access article distributed under the terms and conditions of the Creative Commons Attribution (CC BY) license (http://creativecommons.org/licenses/by/4.0/).

MDPI AG, St. Alban-Anlage 66, 4052 Basel, Switzerland

Peer review is the driving force of journal development, and reviewers are gatekeepers who ensure that Axioms maintains its standards for the high quality of its published papers. Thanks to the cooperation of our reviewers, in 2020, the median time to first decision was 20 days and the median time to publication was 43 days. The editors would like to express their sincere gratitude to the following reviewers for their precious time and dedication, regardless of whether the papers were finally published:

Abreu, Samuel

Abyzov, Adel Nailevich

Acedo, Luis

Acu, Mugur

Afroozeh, Karim

Aguirre-Hernández, Baltazar

Ahmadian, Ali

Akin, Ethan

Alb Lupas, A.

Alcantud, José Carlos

Alexey, Kanel

Alfaro-Garcia, Victor G.

Alzabut, Jehad

Anderson, Douglas

Anghel, Nicolae

Anitescu, Cosmin

Aros, Rodrigo

Artamonov, Viacheslav Alexandrovich

Aslam, Muhammad

Astarita, Vittorio

Aydi, Hassen

Balaz, Antun

Baleanu, Mihaela Cristina

Banakh, Taras

Baranovskii, Evgenii S.

Bardyla, Serhii

Barile, Sergio

Baskonus, Haci Mehmet

Bathrellos, George

Bausys, Romualdas

Bayad, Abdelmejid

Bazighifan, Omar

Becerra Guerrero, Julio

Beckwith, Andrew

Bejenaru, Andreea
Belova, Olga

Binder, Hans

Bobodzhanov, Abdukhafiz

Bognár, Gabriella

Bohaienko, Vsevolod

Boykov, Ilya

Božanić, Darko

Brazas, Jeremy

Brzdek, Janusz

Cai, Zhenning

Cakic, Nenad

Calvaruso, Giovanni

Cao, Bingyang

Cao, Jian

Caponio, Erasmo

Cassese, Gianluca

Casteren, Jan Van

Castro Manzano, Jose Martin

Cattani, Carlo

Čereška, Audrius

Cesarano, Clemente

Chan, Kwokwai

Chang, Chih-Wen

Chasco, M.J.

Chen, Kwang-Wu

Chen, Yanping

Chen, Yi Chou

Chen, Yiyang

Cheng, Qiang

Chino, Marco

Cho, Sun Young

Chow, Kwok W.

Chu, Yu-Ming

Cieplinski, Krzysztof

Cieśliński, Jan L. 
Cirovic, Goran

Concezzi, Moreno

Corda, Christian

Couder-Castañeda, Carlos

Crasmareanu, Mircea

Crutcher, Sihon H.

Cui, Yujun

Curto, Raul

Dao, Son Duy

Das, Pratibhamoy

Dascalescu, Sorin

De La Madrid, Rafael

De La Sen, Manuel

De Swart, Harrie

Debbouche, Amar

Dehmer, Matthias M.

Delcea, Camelia

Deng, Yong

Dey, Arindam

Dix, Julio

Dong, Qiaoli

Dosenovic, Tatjana

Dragomir, Sorin

Dudek, Wieslaw A.

Dvoeglazov, Valeri

Dzedzej, Zdzisław

Egri-Nagy, Attila

El Euch, Hichem

El-Nabulsi, Rami Ahmad

Ernst, Thomas

Etayo, Fernando

Fabregat-Aibar, Laura

Falcón Ganfornina, Raúl Manuel

Faller, Hugues

Farcaseanu, Maria

Favini, Angelo

Fedorov, Vladimir E.

Feltrin, Guglielmo

Fernandez, Arran

Fernandez-Guasti, Manuel

Ferreira, Milton

Fidaleo, Francesco

Fino, Anna Maria

Fish, Andrew

Flouris, Kyriakos

Francisco, Gómez Aguilar José

Fusy, Éric

Gabriela, Cristescu

Gabrielyan, Saak

Gallegos, Javier

Gao, Peichao
García Guirao, Juan

García Salcedo, Ricardo

Garcia, Antonio G.

García-Martínez, Xabier

Garzelli, Maria Vittoria

Georgieva, Atanaska

Germano, Guido

Giambo, Roberto

Gigante, Nicola

Gismatullin, Jakub

Glizer, Valery Y.

Gómez-Aguilar, José Francisco

González Merino, Bernardo

González, Daniel

González-Gaxiola, Oswaldo

Goranko, Valentin

Górniewicz, Lech

Gotchev, Ivan

Grammenos, Theofanis

Grebenisan, Gavril

$\mathrm{Gu}$, Xianming

Guillén, Alberto

Gutik, Oleg

Guze, Sambor

Haba, Zbigniew

Han, Bin

Harirchian, Ehsan

Hayat, Amaury

He, Fei

He, Ji-huan

He, Wei

Hidalgo, Arturo

Hildmann, Hanno

Hofmann, Karl H.

Holighaus, Nicki

Hristova, Snezhana

Hsu, Chang-Hsien

$\mathrm{Hu}$, Lei

Huang, Jinghao

Huang, Tone-Yau

Huertas, Edmundo

Ibrahim, Rabha W.

Ingber, Lester

Ioos, Louis

Ita, Eyo

Jadlovska, Irena

Janno, Jaan

Jiang, Lining

Jordan, Hristov

Jorgensen, Palle E.T.

Julián-Iranzo, Pascual 
Kachalov, Vasiliy

Karapınar, Erdal

Karpinski, Mikołaj

Kazanas, Demosthenes

Khabibullin, Bulat

Khakalo, Sergei

Khater, Mostafa M. A.

Kim, Taekyun

Kim, Yun-Ho

Kirane, Mokhtar

Kok, Chi Wah

Konstantinov, Mihail

Kostić, Marko

Koutras, Athanasios

Koutsogiannis, Andreas

Kuzemsky, Alexander

Kwon, Namhee

Lassoued, Dhaou

Lenzi, Ervin Kaminski

Lewis, Wayne

Li, Changpin

Li, Cunlin

Li, Ming

Li, Tongxing

Li, Xiaowu

Li, Zhiyuan

Liang, Jin

Linsky, Bernard

Liu, Kai

Liu, Yanqin

Liu, Yuanyuan

Lopes, Luiz Guerreiro

Lovyagin, Yuri N.

Lu, Wei

Ludkowski, Sergey

Luisa, D'Amore

Lundström, Niklas L.P.

Magreñán, Ángel Alberto

Malek, Stephane

Marchiafava, Stefano

Mardani, Abbas

Marian, Daniela

Masini, Andrea

Mason, Paolo

Matejička, Ladislav

Matevossian, Hovik

Mehmood, Faizan

Mena-Barboza, Eri A.

Mihai, Adela

Mihály, Bessenyei

Mikeš, Josef
Milanovic, Milos

Miranowicz, Adam

Mironchenko, Andrii

Močkoř, Jiří

Montesinos, Vicente

Moruz, Marilena

Mosavi, Amir

Moysis, Lazaros

Murfet, Daniel

Nakonieczny, Łukasz

Namgung, Ho

Nasibullin, R.

Nian, Jun

Nieto, Juan

Nikolai, Nefedov

Noguera, Carles

Nowak, Piotr

Ogata, Hidenori

Okeke, Godwin Amechi

Ortega, A. Lopez

Ostaszewski, Adam

Paikray, Susanta Kumar

Palatucci, Giampiero

Pamucar, Dragan

Park, C.

Parovik, Roman

Patriciu, Alina-Mihaela

Pauli, Sebastian

Pavlović, Mirjana

Pediconi, Francesco

Peinador, Elena

Pérez, Juan De Dios

Pérez-Domínguez, Luis

Petrukhin, Yaroslav

Pitea, Ariana

Pogrebkov, Andrei

Ponsiglione, Cristina

Popovic, Gabrijela

Povstenko, Yuriy

Pramanik, Tanumoy

Průša, Vít

Prykarpatski, Anatolij

Putinar, Mihai

Qi, Haitao

Qian, Wuyong

Qin, Xiaolong

Rabbi, Fazle

Radenovic, Stojan

Radojevic, Slobodan

Ragulskis, Minvydas

Ragusa, Maria Alessandra 
Rakic, Dusan

Rakshit, Debraj

Ratnikova, Tatjana

Razvarz, Sina

Reich, Simeon

Riedel, Thomas

Robert, Frédéric

Rosas-Ortiz, Oscar

Sababe, Saeed

Salimi, Mehdi

Sanchez-Ortiz, Jorge

Sanchez-Soto, Luis

Sandev, Trifce

Sándor, Bulcsú

Savin, Diana

Sawa, Koji

Scapellato, Andrea

Schaeffer, Satu Elisa

Sebastião, Helder

Semenov, Vladimir V.

Sene, Ndolane

Sergi, Pier Nicola

Sessa, Salvatore

Shalyt-Margolin, Alexander

Shang, Yilun

Shapovalov, Alexander

Sharifi, Somayeh

Sharifov, Yagub

Shi, Fu-Gui

Shin, Dong Yun

Shin, M. Minsuk

Sidorov, Denis

Simon, Istvan

Soares, Junior Cesar Alves

Solym M., MANOU- ABI

Song, Chunyue

Sostak, Alexander

Souayah, Nizar

Srivastava, H. M.

Stacho, Laszlo

Sun, Huafei

Syropoulos, Apostolos

Takahashi, Futoshi

Tamariz Mascarúa, Ángel

Taniguchi, Shigeru

Tas, Nihal

Tasche, Manfred

Tedder, Andrew

Temuer, Chaolu
Teofanov, Nenad

Tkachenko, Michael

Todorčević, Vesna

Treanta, Savin

Treesatayapun, Chidentree

Trinh, Minh-Chien

Triolo, Salvatore

Tsekhan, Olga

Turcanu, Teodor

Turinici, Mihai

Tutueva, Aleksandra

Ullah, Kifayat

Uppulury, Karthik

Usurelu, Gabriela Ioana

Van Der Veken, Joeri

Van Mill, Jan

Verma, Rajkumar

Versaci, Mario

Voigtländer, Felix

Volinsky, Irina

Vosika, Zoran

Wang, Bin

Wang, Chao

Wang, Jun

Wang, Sanmin

Wang, Zhiqiang

Weber, Eric S.

Wei, Yiheng

$\mathrm{Wu}$, Guocheng

Xiao, Dunhui

Xie, Jiafeng

Xiong, Hao

Yang, Zhan-Peng

Yang, ZhenBiao

Yao, Jen-Chih

Yao, Yonghong

Yavuz, Mehmet

Yukalov, V.I.

Yuldashev, Tursun

Zagorodnyuk, Andriy

Zambrano-Serrano, Ernesto

Zeifman, Alexander

Zhang, Fu

Zhang, Yanpeng

Zhang, Zhen

Zhao, Ying

Zhao, Zhenyu

Zimmermann, Rainer E.

Żur, Krzysztof Kamil 